\title{
A New Reduced Stabilized Mixed Finite-Element Method Based on Proper Orthogonal Decomposition for the Transient Navier-Stokes Equations
}

\author{
Aiwen Wang, ${ }^{1}$ Jian Li, $^{2}$ Zhenhua Di, $^{3}$ Xiangjun Tian, ${ }^{4}$ \\ and Dongxiu Xie ${ }^{1}$ \\ ${ }^{1}$ School of Applied Science, Beijing Information Science and Technology University, Beijing 100192, China \\ ${ }^{2}$ Department of Mathematics, Baoji University of Arts and Sciences, Baoji 721007, China \\ ${ }^{3}$ College of Global Change and Earth System Science, Beijing Normal University, Beijing 100009, China \\ ${ }^{4}$ Institute of Atmospheric Physics, Chinese Academy of Sciences, Beijing 100029, China
}

Correspondence should be addressed to Aiwen Wang, wawjianmo@126.com

Received 31 May 2011; Accepted 15 August 2011

Academic Editor: Jan Sladek

Copyright (c) 2011 Aiwen Wang et al. This is an open access article distributed under the Creative Commons Attribution License, which permits unrestricted use, distribution, and reproduction in any medium, provided the original work is properly cited.

\begin{abstract}
A reduced stabilized mixed finite-element (RSMFE) formulation based on proper orthogonal decomposition (POD) for the transient Navier-Stokes equations is presented. An ensemble of snapshots is compiled from the transient solutions derived from a stabilized mixed finite-element (SMFE) method based on two local Gauss integrations for the two-dimensional transient NavierStokes equations by using the lowest equal-order pair of finite elements. Then, the optimal orthogonal bases are reconstructed by implementing POD techniques for the ensemble snapshots. Combining POD with the SMFE formulation, a new low-dimensional and highly accurate SMFE method for the transient Navier-Stokes equations is obtained. The RSMFE formulation could not only greatly reduce its degrees of freedom but also circumvent the constraint of inf-sup stability condition. Error estimates between the SMFE solutions and the RSMFE solutions are derived. Numerical tests confirm that the errors between the RSMFE solutions and the SMFE solutions are consistent with the the theoretical results. Conclusion can be drawn that RSMFE method is feasible and efficient for solving the transient Navier-Stokes equations.
\end{abstract}

\section{Introduction}

Mixed finite-element (MFE) methods are one of the most important approaches for solving the nonstationary Navier-Stokes equations [1-3]. However, some fully discrete MFE formulations for the nonstationary Navier-Stokes equations involve generally many degrees of freedom. In addition, the importance of ensuring the compatibility of the approximations 
for the velocity and pressure by satisfying the so-called inf-sup condition (LBB condition) is widely understood. Thus, an important problem is how to avoid the lack of the LBB stability and simplify the computational load by saving time-consuming calculations and resource demands in the actual computational process in a way that guarantees a sufficiently accurate numerical solution.

Proper orthogonal decomposition (POD) is an effective method for approximating a large amount of data. The method essentially finds a group of orthogonal bases from the given data to approximately represent them in a least squares optimal sense. In addition, as the POD is optimal in the least squares sense, it has the property that the model decomposition is completely dependent on the given data and does not require assuming any prior knowledge of the process. Combined with a Galerkin projection procedure, POD provides a powerful method for deriving lower dimensional models of dynamical systems from a high or even infinite dimensional space. A dynamic system is generally governed by related structures or the ensemble formed by a large number of different instantaneous solutions, and the POD method can capture the temporal and spatial structures of dynamic system by applying a statistical analysis to the ensemble of data. POD provides an adequate approximation for a large amount of data with a reduced number of degrees of freedom; it alleviates the computational load and provides substantial savings in memory requirements. POD has found widespread application in a variety of fields such as signal analysis and pattern recognition $[4,5]$, fluid dynamics and coherent structures [6-8], optimal flow control problems $[9,10]$, and land surface soil moisture data assimilation [11]. In fluid dynamics, Lumley first applied the POD method to capture the large eddy coherent structures in a turbulent boundary layer [12]. This method was further applied to study the relation between the turbulent structure and a chaotic dynamic system [13]. Sirovich introduced the method of snapshots and applied it to reduce the order of POD eigenvalue problem [14]. Kunisch and Volkwein presented Galerkin POD methods for parabolic problems and a general equation in fluid dynamics $[15,16]$. More recently, a finite difference scheme (FDS) and a MFE formulation for the nonstationary Navier-Stokes equation based on POD were derived [17, 18], respectively. Finite-element formulation based on POD was also applied for parabolic equations and the Burgers equation [19, 20]. In other physical applications, an effective use of POD for a chemical vapor deposition reactor was demonstrated and some reduced-order FDS and MFE for the upper tropical Pacific Ocean model based on POD were presented [21-25]. An optimizing reduced FDS based on POD for the chemical vapor deposit (CVD) equations was also presented in [26]. Except for POD, the empirical orthogonal function (EOF) analysis is another effective method to extract information from large datasets in time and space $[27,28]$.

In order to avoid the lack of LBB stability, some kinds of stabilized techniques for the lowest-order finite elements appear in [29-44]. Luo et al.[45] has combined the POD method with a stabilize method [40] to deal with the non-stationary Navier-Stokes equations and obtained good results. But the stabilized mixed methods in $[40,45]$ are often developed using residuals of the momentum equation. These residual terms must be formulated using meshdependent parameters, whose optimal values are usually unknown. Particularly, for the lowest equal-order pairs of mixed elements such as $P_{1}-P_{1}$ and $Q_{1}-Q_{1}$, pressure and velocity derivatives in the residual either vanish or are poorly approximated, causing difficulties in the application of consistent stabilization.

In this paper, we mainly consider the two-dimensional transient Navier-Stokes equations by combining a new stabilized finite-element method [29-31] based on two local Gauss' integrations with POD method. This new stabilized finite element method has some 
prominent features: parameter-free, avoiding higher-order derivatives or edge-based data structures, and stabilization being completed locally at the element level. In this manner, we could not only ensure the stabilization of solutions of fully discrete stabilized mixed finite-element system but also greatly reduce degrees of freedom and save time-consuming calculations and resource demands in the actual computational process in a way that guarantees a sufficiently accurate numerical solution. we also derive the error estimates between the original SMFE solutions and the RSMFE solutions based on the POD technique. Numerical experiments show the errors between the original SMFE method and the RSMFE solutions are consistent with theoretical results.

The remainder of this paper is organized as follows. In Section 2, an abstract functional setting for the two-dimensional Navier-Stokes equations is given, together with some basic notations. Section 3 is to state the fully discrete stabilized finite-element method and to generate snapshots from transient solutions computed from the equation system derived by the classical SMFE formulation. In Section 4, the optimal orthogonal bases are reconstructed from the elements of the snapshots with POD method and a reduced SMFE formulation with lower-dimensional number based on POD method for the transient Navier-Stokes equations is developed. In Section 5, error estimates between the classical SMFE solutions and the RSMFE solutions based on the POD method are derived. In Section 6, a series of numerical experiments are given to illustrate the theoretical results. We conclude with a few remarks in the final section.

\section{Functional Setting of the Navier-Stokes Equations}

Let $\Omega$ be a bounded domain in $R^{2}$, assumed to have a Lipschitz continuous boundary $\Gamma$ and to satisfy further assumptions below. The transient Navier-Stokes equations are considered as follows:

$$
\begin{gathered}
u_{t}-v \Delta u+(u \cdot \nabla) u+\nabla p=f, \quad \operatorname{div} u=0, \quad(x, t) \in \Omega \times(0, T], \\
u(x, 0)=u_{0}(x), \quad x \in \Omega,\left.\quad u(x, t)\right|_{\Gamma}=0, \quad t \in[0, T] .
\end{gathered}
$$

Here $u: \Omega \rightarrow R^{2}$ and $p: \Omega \rightarrow R$ are the velocity and pressure, $v>0$ is the viscosity, and $f$ represents the body forces, $T>0$ the final time, and $u_{t}=\partial u / \partial t$.

For the mathematical setting of problems (2.1)-(2.2), we introduce the following Sobolev spaces:

$$
\begin{gathered}
X=H_{0}^{1}(\Omega)^{2}, \quad M=L_{0}^{2}(\Omega)=\left\{q \in L^{2}(\Omega) ; \int_{\Omega} q(x) d x=0\right\}, \\
D(A)=H^{2}(\Omega)^{2} \cap X, \quad V=\{v \in V: \operatorname{div} v=0\} .
\end{gathered}
$$

Furthermore, we make a regularity assumption on the Stokes problem as follows.

Assumption H1. For a given $g \in Y$ and the Stokes problem,

$$
\begin{aligned}
-\Delta v+\nabla q=g, & \text { in } \Omega, \\
\operatorname{div} v=0, & \text { in } \Omega, \\
\left.v\right|_{\Gamma}=0, & \text { on } \partial \Omega,
\end{aligned}
$$


satisfying the following regularity result:

$$
\|v\|_{2}+\|q\|_{1} \leq \kappa\|g\|_{0}
$$

where $\|\cdot\|_{i}$ is the norm of the Sobolev space $H^{i}(\Omega)$ or $H^{i}(\Omega)^{2}, i=0,1,2$, as appropriate, and $\kappa$ is a positive constant depending only on $\Omega$, which may stand for different value at its different occurrences. Subsequently, the positive constants $\mathcal{K}$ and $c$ (with or without a subscript) will depend only on the data $\left(\nu, T, \Omega, u_{0}\right)$. Because the norm and seminorm are equivalent on $H_{0}^{1}(\Omega)^{2}$, we use the same notation $\|\cdot\|_{1}$ for them. It is well known that for each $v \in X$ there hold the following inequalities:

$$
\|v\|_{L^{4}} \leq 2^{1 / 4}\|v\|_{0}^{1 / 2}\|v\|_{1}^{1 / 2}
$$

Assumption H2. The initial velocity $u_{0} \in D(A)$ and the body force $f(x, t) \in L^{2}\left(0, T ; L^{2}(\Omega)^{2}\right)$ are assumed to satisfy

$$
\left\|u_{0}\right\|_{2}+\left(\int_{0}^{T}\left(\|f\|_{0}^{2}+\left\|f_{t}\right\|_{0}^{2}\right) d t\right)^{1 / 2} \leq c
$$

Now, the bilinear forms $a(\cdot, \cdot)$ and $d(\cdot, \cdot)$, on $X \times X$ and $X \times M$, are defined, respectively, by

$$
a(u, v)=v(\nabla u, \nabla v), \quad \forall u, v \in X, \quad d(v, q)=(q, \operatorname{div} v), \quad \forall(v, q) \in(X, M)
$$

Also, a generalized bilinear form $B((\cdot, \cdot) ;(\cdot, \cdot))$ on $(X, M) \times(X, M)$ is defined by

$$
B((u, p) ;(v, q))=a(u, v)-d(v, p)+d(u, q)
$$

Moreover, we define the trilinear form

$$
\begin{aligned}
b(u, v, w) & =((u \cdot \nabla) v, w)+\frac{1}{2}((\operatorname{div} u) v, w) \\
& =\frac{1}{2}((u \cdot \nabla) v, w)-\frac{1}{2}((u \cdot \nabla) w, v), \quad \forall u, v, w \in X
\end{aligned}
$$

By the above notations and the Hölder inequality, there hold the following estimates:

$$
\begin{gathered}
b(u, v, w)=-b(u, w, v), \quad \forall u \in X, v, w \in X, \\
|b(u, v, w)| \leq \frac{1}{2} c_{0}\|u\|_{0}^{1 / 2}\|u\|_{1}^{1 / 2}\left(\|v\|_{1}\|w\|_{0}^{1 / 2}\|w\|_{1}^{1 / 2}+\|v\|_{0}^{1 / 2}\|v\|_{1}^{1 / 2}\|w\|_{1}\right), \quad \forall u, v, w \in X, \\
|b(u, v, w)|+|b(v, u, w)|+|b(w, u, v)| \leq c_{1}\|u\|_{1}\|v\|_{2}\|w\|_{0}, \quad \forall u \in X, v \in D(A), w \in Y .
\end{gathered}
$$


Also, the Poincare inequality holds:

$$
\|v\|_{0} \leq \gamma_{0}\|v\|_{1}
$$

where $c_{0}, c_{1}$, and $\gamma_{0}$ are positive constants depending only on $\Omega$.

For a given $f \in Y$, the variational formulation of problem (2.1)-(2.2) reads as follows: find $(u, p) \in(X, M), t>0$ such that

$$
\begin{gathered}
\left(u_{t}, v\right)+\mathcal{B}((u, p) ;(v, q))+b(u, u, v)=(f, v), \quad \forall(v, q) \in(X, M), \\
u(0)=u_{0} .
\end{gathered}
$$

For convenience, we recall the discrete Gronwall Lemma that will be frequently used.

Lemma 2.1 (see $[1,45,46])$. Let $\left\{a_{n}\right\},\left\{b_{n}\right\}$, and $\left\{c_{n}\right\}$ be three positive sequences, and let $\left\{c_{n}\right\}$ be monotone and satisfy

$$
a_{n}+b_{n} \leq c_{n}+\lambda \sum_{i=0}^{n-1} a_{i}, \quad l>0, \quad a_{0}+b_{0} \leq c_{0}
$$

then

$$
a_{n}+b_{n} \leq c_{n} \exp (n \lambda), \quad n \geq 0 .
$$

The following existence and uniqueness result is classical (see [1, 46]).

Theorem 2.2. Assume that (H1) and (H2) hold. Then, for any given $T>0$, there exists a unique solution $(u, p)$ satisfying the following regularities:

$$
\begin{gathered}
\sup _{0<t \leq T}\left(\|u(t)\|_{2}^{2}+\|p(t)\|_{1}^{2}+\left\|u_{t}(t)\right\|_{0}^{2}\right) \leq c, \\
\sup _{0<t \leq T} \tau(t)\left\|u_{t}\right\|_{1}^{2}+\int_{0}^{T} \tau(t)\left(\left\|u_{t}\right\|_{2}^{2}+\left\|p_{t}\right\|_{1}^{2}+\left\|u_{t t}\right\|_{0}^{2}\right) d t \leq c,
\end{gathered}
$$

where $\tau(t)=\min \{1, t\}$.

\section{Fully Discrete SMFE Method and Generation of Snapshots}

In this section, we focus on the stabilized method proposed by [29] for the Stokes equations. Let $h>0$ be a real positive parameter. Finite-element subspace $\left(X_{h}, M_{h}\right)$ of $(X, M)$ is characterized by $\tau_{h}=\tau_{h}(\Omega)$, a partitioning of $\bar{\Omega}$ into triangles or quadrilaterals $K$, assumed to be regular in the usual sense; that is, for some $\sigma$ and $\omega$ with $\sigma>1$ and $0<\omega<1$,

$$
\begin{gathered}
h_{K} \leq \sigma \rho_{K}, \quad \forall K \in \tau_{h} \\
\left|\cos \theta_{i K}\right| \leq \omega, \quad i=1,2,3,4, \quad \forall K \in \tau_{h}
\end{gathered}
$$


where $h_{K}$ is the diameter of element $K, \rho_{K}$ is the diameter of the inscribed circle of element $K$, and $\theta_{i K}$ are the angles of $K$ in the case of a quadrilateral partitioning. The mesh parameter $h$ is given by $h=\max _{k \in \tau_{h}} h_{K}$. The finite-element subspaces of this paper are defined by setting

$$
R_{1}(K)= \begin{cases}P_{1}(K), & \text { if } \mathrm{K} \text { is triangular } \\ Q_{1}(K), & \text { if } \mathrm{K} \text { is the quadrilateral }\end{cases}
$$

Then, the finite-element pairs are coupled as follows:

$$
\begin{aligned}
& X_{h}=\left\{v \in X ;\left.v_{i}\right|_{K} \in R_{1}(K), \quad i=1,2\right\}, \\
& M_{h}=\left\{q \in M:\left.q\right|_{K} \in R_{1}(K), \forall K \in \tau_{h}\right\} .
\end{aligned}
$$

It is well known that this lowest equal-order finite-element pair does not satisfy the infsup condition. We define the following local difference between a consistent and an underintegrated mass matrices the stabilized formulation [29-31]:

$$
G\left(p_{h}, q_{h}\right)=p_{i}^{T}\left(M_{k}-M_{1}\right) q_{j}=p_{i}^{T} M_{k} q_{j}-p_{i}^{T} M_{1} q_{j}
$$

Here, we set

$$
\begin{gathered}
p_{i}^{T}=\left[p_{0}, p_{1}, \ldots, p_{N-1}\right]^{T}, \quad q_{j}=\left[q_{0}, q_{1}, \ldots, q_{N-1}\right], \\
M_{i j}=\left(\phi_{i}, \phi_{j}\right), \quad p_{h}=\sum_{i=0}^{N-1} p_{i} \phi_{i} \\
p_{i}=p_{h}\left(x_{i}\right), \quad \forall p_{h} \in M_{h}, i, j=0, \ldots, N-1,
\end{gathered}
$$

where $\phi_{i}$ is the basis function of the pressure on the domain $\Omega$ such that its value is one at node $x_{i}$ and zero at other nodes; the symmetric and positive $M_{k}, k \geq 2$ and $M_{1}$ are pressure mass matrix computed by using the k-order and 1-order Gauss integrations in each direction; respectively, also, $p_{i}$ and $q_{i}, i=0,1, \ldots, N$, are the value of $p_{h}$ and $q_{h}$ at the node $x_{i} . p_{i}^{T}$ is the transpose of the matrix $p_{i}$.

Let $\Pi_{h}: M \rightarrow R_{0}$ be the standard $L^{2}$-projection with the following properties [29-32]:

$$
\begin{gathered}
\left(p, q_{h}\right)=\left(\Pi_{h} p, q_{h}\right), \quad \forall p \in M, q_{h} \in R_{0}, \\
\left\|\Pi_{h} p\right\|_{0} \leq c\|p\|_{0}, \quad \forall p \in M, \\
\left\|p-\Pi_{h} p\right\|_{0} \leq\|p\|_{1}, \quad \forall p \in H^{1}(\Omega) \cap M,
\end{gathered}
$$

where $R_{0}=\left\{q_{h} \in M:\left.q_{h}\right|_{K}\right.$ is a constant, $\left.\forall K \in K_{h}\right\}$. Then we can rewrite the bilinear form $G(\cdot, \cdot)$ by

$$
G(p, q)=(p-\Pi p, q-\Pi q)
$$


Remark 3.1. The bilinear form $G(\cdot, \cdot)$ in (3.7) is a symmetric, semipositive definite form generated on each local set $K$. The term can alleviate and offset the inf-sup condition [29]. It differs from the stabilized term in [45]. It does not require a selection of mesh-dependent stabilization parameters or a calculation of higher-order derivatives. Its another valuable feature is that the action of stabilization operators can be performed locally at the element level with minimal additional cost.

With the above notation, we begin by choosing an integer $N$ and defining the time step $\tau=T / N$ and discrete times $t_{n}=n \tau, n=0,1,2, \ldots, N$. We obtain the fully discrete scheme as follows: find functions $\left\{u_{h}^{n}\right\}_{n \geq 0} \subset X_{h}$ and $\left\{p_{h}^{n}\right\}_{n \geq 1} \subset M_{h}$ as solutions of the recursive linear algebraic equations,

$$
\begin{gathered}
\left(d_{t} u_{h}^{n}, v_{h}\right)+B_{h}\left(\left(u_{h}^{n}, p_{h}^{n}\right) ;\left(v_{h}, q_{h}\right)\right)+b\left(u_{h}^{n-1}, u_{h}, v_{h}\right)=\left(f\left(t_{n}\right), v_{h}\right), \\
u_{h}^{0}=u_{0 h} .
\end{gathered}
$$

for all $\left(v_{h}, q_{h}\right) \in\left(X_{h}, M_{h}\right)$, where

$$
\begin{gathered}
d_{t} u_{h}^{n}=\frac{u_{h}^{n}-u_{h}^{n-1}}{\tau}, \\
B_{h}\left(\left(u_{h}, p_{h}\right) ;\left(v_{h}, q_{h}\right)\right)=a\left(u_{h}, v_{h}\right)-d\left(v_{h}, p_{h}\right)+d\left(u_{h}, q_{h}\right)+G\left(p_{h}, q_{h}\right),
\end{gathered}
$$

and $u_{0 h}$ is the approximation of $u_{0}$. The solutions $\left\{u_{h}^{n}\right\}_{n \geq 0}$ and $\left\{\bar{p}_{h}^{n}\right\}_{n \geq 1}$ to (3.8)-(3.9) are expected to the approximations of $\left\{u_{h}\left(t_{n}\right)\right\}_{n \geq 0}$ and $\left\{\bar{p}_{h}\left(t_{n}\right)\right\}_{n \geq 1}$ with

$$
\bar{p}_{h}\left(t_{n}\right)=\frac{1}{\tau} \int_{t_{n-1}}^{t_{n}} p_{h}(t) d t
$$

Theorem 3.2 (see [29-32]). Let $\left(X_{h}, M_{h}\right)$ be defined as above, then there exists a positive constant $\beta$, independent of $h$, such that

$$
\begin{gathered}
|\mathbb{B}((u, p) ;(v, q))| \leq c\left(\|u\|_{1}+\|p\|_{0}\right)\left(\|v\|_{1}+\|q\|_{0}\right), \quad(u, p),(v, q) \in(X, M), \\
\beta\left(\left\|u_{h}\right\|_{1}+\left\|p_{h}\right\|_{0}\right) \leq \sup _{\left(v_{h}, q_{h}\right) \in\left(X_{h}, M_{h}\right)} \frac{\left|B_{h}\left(\left(u_{h}, p_{h}\right) ;\left(v_{h}, q_{h}\right)\right)\right|}{\left\|v_{h}\right\|_{1}+\left\|q_{h}\right\|_{0}}, \quad \forall\left(u_{h}, p_{h}\right) \in\left(X_{h}, M_{h}\right), \\
|G(p, q)| \leq c\left\|p-\Pi_{h} p\right\|_{0}\left\|q-\Pi_{h} q\right\|_{0^{\prime}} \quad \forall p, q \in M .
\end{gathered}
$$

By using the same approaches as those in [45], we can prove the following result.

Theorem 3.3. Under the assumptions of Theorems 2.2 and 3.2, if $h$ and $\tau$ are sufficiently small and $h=O(\tau)$, then problem (3.8)-(3.9) has a unique solution $\left(u_{h^{\prime}}^{n} p_{h}^{n}\right) \in X_{h} \times M_{h}$ such that

$$
\left\|u_{h}^{n}\right\|_{0}^{2}+\sum_{j=1}^{n}\left(\tau \mathcal{v}\left\|\nabla u_{h}^{j}\right\|_{0}^{2}+\tau G\left(p_{h^{\prime}}^{j} p_{h}^{j}\right)\right) \leq\left\|u_{0}\right\|_{0}^{2}+c \tau v^{-1} \sum_{j=1}^{n}\left\|f^{j}\right\|_{0}^{2} .
$$


Theorem 3.4 (see [32]). Under the assumptions of Theorem 3.3, the error $\left(u\left(t_{n}\right)-u_{h^{\prime}}^{n} p\left(t_{n}\right)-p_{h}^{n}\right)$ satisfies the following bound:

$$
\begin{gathered}
\tau \sum_{n=1}^{N}\left\|u\left(t_{n}\right)-u_{h}^{n}\right\|_{0}^{2}+\tau\left(t_{m}\right)\left\|u\left(t_{m}\right)-u_{h}^{m}\right\|_{0}^{2} \leq c\left(h^{4}+\tau^{2}\right), \\
\tau \sum_{n=1}^{N}\left\|u\left(t_{n}\right)-u_{h}^{n}\right\|_{1}^{2}+\tau\left(t_{m}\right)\left\|u\left(t_{m}\right)-u_{h}^{m}\right\|_{1}^{2} \leq c\left(h^{2}+\tau^{2}\right), \\
\tau \sum_{n=1}^{N} \tau\left(t_{n}\right)\left\|p\left(t_{n}\right)-p_{h}^{n}\right\|_{0}^{2} \leq c\left(h^{2}+\tau^{2}\right),
\end{gathered}
$$

for all $t_{m} \in(0, T]$.

If $v$, the time step increment $\tau$, and the right-hand side $f$ are given, by solving problem(3.8)(3.9), we can obtain solution ensemble $\left\{u_{1 h^{\prime}}^{n} u_{2 h^{\prime}}^{n} p_{h}^{n}\right\}_{n=1}^{L}$. Then we choose $L$ (in general, $L \leq N$, e.g., $L=20, N=200)$ instantaneous solutions $U_{i}(x, y)=\left(u_{1 h^{\prime}}^{i} u_{2 h^{\prime}}^{i} p_{h}^{i}\right)(i=1,2, \ldots, L)$ from the $L$ group of solutions $\left(u_{h}^{n}, p_{h}^{n}\right)(1 \leq n \leq L)$ for problems (3.8), which are known as snapshots.

Remark 3.5. When one computes actual problems, one may obtain the ensemble of snapshots from physical system trajectories by drawing samples from experiments and interpolation (or data assimilation). For example, when one finds numerical solutions to PDES representing weather forecast, one can use the previous weather prediction results to construct the ensemble of snapshots, then restructure the POD optimal basis for the ensemble of snapshots by the following POD method, next replace finite element space $\left(X_{h}, M_{h}\right)$ with the subspace spanned by the optimal POD basis. Numerical weather forecast equation is reduced to a fully discrete algebra equation with fewer degrees of freedom. Thus, the forecast of future weather change can be quickly simulated, which is a result of major importance for real-life applications.

\section{Reduced SMFE Formulation Based on POD Method}

The POD method has received much attention in recent years as a tool to analyze complex physical systems. In this section, we use POD technique to deal with the snapshots in Section 3 and then use the POD basis to develop an RSMFE formulation for the transient Navier-Stokes equations.

Let $\widehat{X}=X \times M$, and let $U_{i}(x, y)=\left(u_{1 h^{\prime}}^{i}, u_{2 h^{\prime}}^{i} p_{h}^{i}\right)(i=1,2, \ldots, L$, see Section 3$)$. Set

$$
V=\operatorname{span}\left\{U_{1}, U_{2}, \ldots, U_{L}\right\},
$$

where $V$ is the ensemble consisting of the snapshots $\left\{U_{i}\right\}_{i=1}^{L}$, at least one of which is supposed to nonzero. Let $\left\{\Psi_{j}\right\}_{j=1}^{l}$ denote an orthogonal basis of $V$ with $l=\operatorname{dim} V(l \leq L)$. Then each member of the ensemble is expressed as

$$
U_{i}=\sum_{i=1}^{l}\left(U_{i}, \Psi_{j}\right)_{\widehat{X}} \Psi_{j}, \quad \text { for } i=1,2, \ldots, L,
$$

where $\left(U_{i}, \Psi_{j}\right)_{\hat{X}}=\left(u_{h}^{i}, \Psi_{u j}\right)+\left(p_{h}^{i}, \Psi_{p j}\right), \Psi_{u j}$ and $\Psi_{p j}$ are the orthogonal basis corresponding to $u$ and $p$, respectively. 
The method of POD consists in finding the orthogonal basis such that, for every $d(1 \leq$ $d \leq L)$, the mean square error between the elements $U_{i}(1 \leq i \leq L)$ and corresponding $d$ th partial sum of (4.2) is minimized on average

$$
\min _{\left\{\Psi_{j}\right\}_{j=1}^{d}} \frac{1}{L} \sum_{i=1}^{L}\left\|U_{i}-\sum_{j=1}^{d}\left(U_{i}, \Psi_{j}\right)_{\widehat{X}} \Psi_{j}\right\|_{\widehat{X}}^{2},
$$

subject to

$$
\left(\Psi_{i}, \Psi_{j}\right)_{\hat{X}}=\delta_{i j}, \quad \text { for } 1 \leq i \leq d, \quad 1 \leq j \leq i
$$

where $\left\|U_{i}\right\|_{\hat{X}}=\left(\left\|u_{1 h}^{i}\right\|_{1}^{2}+\left\|u_{2 h}^{i}\right\|_{1}^{2}+\left\|p_{h}^{i}\right\|_{0}^{2}\right)^{1 / 2}$. A solution sequence $\left\{\Psi_{j}\right\}_{j=1}^{d}$ of $(4.3)$ and $(4.4)$ is known as a POD basis of rank $d$.

We introduce the correlation matrix $E=\left(E_{i j}\right)_{L \times L} \in R^{L \times L}$ corresponding to the snapshots $\left\{U_{i}\right\}_{i=1}^{L}$ by

$$
E_{i j}=\frac{1}{L}\left(U_{i}, U_{j}\right)_{\hat{X}}
$$

The matrix $E$ is positive semidefinite and has rank $l$. The solution of (4.3) and (4.4) can be found in [45].

Proposition 4.1. Let $\lambda_{1} \geq \lambda_{2} \geq \cdots \geq \lambda_{l}>0$ denote the positive eigenvalues of $E$, and let $v_{1}, v_{2}, \ldots, v_{l}$ be the associated eigenvectors. Then a POD basis of rank $d \leq l$ is given by

$$
\Psi_{i}=\frac{1}{\sqrt{\lambda_{i}}} \sum_{j=1}^{L}\left(v_{i}\right)_{j}, U_{j}, \quad i=1,2, \ldots, d \leq l,
$$

where $\left(v_{i}\right)_{j}$ denotes the $j$ th component of the eigenvector $v_{i}$. Furthermore, the following error formula holds:

$$
\frac{1}{L} \sum_{i=1}^{L}\left\|U_{i}-\sum_{j=1}^{d}\left(U_{i}, \Psi_{j}\right)_{\hat{X}} \Psi_{j}\right\|_{\hat{X}}^{2}=\sum_{j=d+1}^{l} \lambda_{j}
$$

Let $V^{d}=\left\{\Psi_{1}, \Psi_{2}, \ldots, \Psi_{d}\right\}$, and let $X^{d} \times M^{d}=V^{d}$ with $X^{d} \subset X_{h} \subset X$, and let $M^{d} \subset M_{h} \subset M$. Set the Ritz-projection $P^{h}: X \rightarrow X_{h}$ (if $P^{h}$ is restricted to the Ritz-projection from $X_{h}$ to $X^{d}$, it is written as $P^{d}$ ) such that $\left.P^{h}\right|_{X_{h}}=P^{d}: X_{h} \rightarrow X^{d}$ and $P^{h}: X \backslash X_{h} \rightarrow X_{h} \backslash X^{d}$ and $L^{2}$-projection $\rho^{d}: M \rightarrow M^{d}$ denoted by, respectively,

$$
\begin{gathered}
a\left(P^{h} u, v_{h}\right)=a\left(u, v_{h}\right), \quad \forall v_{h} \in X_{h}, \\
\left(\rho^{d} p, q_{d}\right)_{0}=\left(p, q_{d}\right)_{0}, \quad q_{d} \in M^{d},
\end{gathered}
$$

where $u \in X$ and $p \in M$. Owing to (4.8)-(4.9) the linear operators $P^{h}$ and $\rho^{d}$ are well defined and bounded:

$$
\left\|P^{d} u\right\|_{1} \leq\|u\|_{1}, \quad\left\|\rho^{d} p\right\|_{0^{\prime}} \leq\|p\|_{0^{\prime}} \quad \forall u \in X, \quad p \in M
$$


Lemma 4.2 (see [45]). For every $d(1 \leq d \leq l)$, the projection operators $P^{d}$ and $\rho^{d}$ satisfy, respectively,

$$
\begin{gathered}
\frac{1}{L} \sum_{i=1}^{L}\left\|u_{h}^{n_{i}}-P^{d} u_{h}^{n_{i}}\right\|_{1}^{2} \leq \sum_{j=d+1}^{l} \lambda_{j}, \\
\frac{1}{L} \sum_{i=1}^{L}\left\|u_{h}^{n_{i}}-P^{d} u_{h}^{n_{i}}\right\|_{0}^{2} \leq C h^{2} \sum_{j=d+1}^{l} \lambda_{j}, \\
\frac{1}{L} \sum_{i=1}^{L}\left\|p_{h}^{n_{i}}-\rho^{d} p_{h}^{n_{i}}\right\|_{0}^{2} \leq \sum_{j=d+1}^{l} \lambda_{j},
\end{gathered}
$$

where $u_{h}^{n_{i}}=\left(u_{1 h}^{n_{i}}, u_{2 h}^{n_{i}}\right)$ and $\left(u_{1 h^{\prime}}^{n_{i}}, u_{2 h}^{n_{i}}, p_{h}^{n_{i}}\right) \in V$.

Thus, using $V^{d}=X^{d} \times M^{d}$, we can obtain the reduced SMFE formulation for problems (3.8) as follows. Find $\widehat{u}_{d}^{n}=\left(u_{d^{\prime}}^{n} p_{d}^{n}\right) \in V^{d}$ such that

$$
\begin{gathered}
\left(d_{t} u_{d}^{n}, v_{d}\right)+B_{h}\left(\left(u_{d}^{n}, p_{d}^{n}\right) ;\left(v_{d}, q_{d}\right)\right)+b\left(u_{d}^{n-1}, u_{d}, v_{d}\right)=\left(f\left(t_{n}\right), v_{d}\right), \quad 1 \leq n \leq N, \\
u_{d}^{0}=u_{0 h} .
\end{gathered}
$$

Remark 4.3. Problem (3.8) includes $N_{h}\left(N_{h}\right.$ is the number of triangles or quadrilaterals vertex in $\tau_{h}$ ) freedom degrees, while problem (4.14) includes $d(d \ll l \leq L \leq N)$ freedom degrees. For actual science and engineering problems, the number of the vertex in $\tau_{h}$ are tens of thousands, even hundreds of millions, but $d$ is the number of the largest eigenvalues of $l$ snapshots from $L$ transient solutions; it is very small. For numerical example in Section 6, $d=7$, but $N_{h}=32 \times 32 \times 3=3072$. Thus, problem (4.14) is a simplified stabilized finiteelement scheme. In addition, the future development of many natural phenomena is affected by previous information, such as biological evolution and weather change. Here, we use the existing data to construct the POD basis, which contains the information on past data. Thus, this method can not only save computational load, but also make better use of the existing information to capture the law of the future development of natural phenomena.

\section{Existence and Error Analysis of Solution to the Optimizing RSMFE Formulation}

This section is devoted to discussing the existence and error estimates of solutions to problem (4.14). We see from (4.6) that $V^{d}=X^{d} \times M^{d} \subset V \subset X_{h} \times M_{h} \subset X \times M$. Using the same approaches as proving Theorem 3.3, we could prove the following existence result for solutions of problem (4.14).

Theorem 5.1. Under the assumptions of Theorems 2.2 and 3.3, Problem (4.14) has a unique solution sequence $\left(u_{d^{\prime}}^{n}, p_{d}^{n}\right) \in X^{d} \times M^{d}$ and satisfies, for $1 \leq n \leq N$,

$$
\left\|u_{d}^{n}\right\|_{0}^{2}+\sum_{j=1}^{n}\left(\tau v\left\|u_{d}^{j}\right\|_{1}^{2}+\tau G\left(p_{d}^{j}, p_{d}^{j}\right)\right) \leq\left\|u_{0}\right\|_{0}^{2}+c \tau v^{-1} \sum_{j=1}^{n}\left\|f^{j}\right\|_{0}^{2}
$$

In the following theorem, the errors between the solution $\left(u_{d^{\prime}}^{n} p_{d}^{n}\right)$ to Problem (4.14)(4.15) and the solution $\left(u_{h}^{n}, p_{h}^{n}\right)$ to Problem (3.8) are derived. 
Theorem 5.2. Under the assumptions of Theorem 5.1, if $h$ and $\tau$ are sufficiently small, $h=O(\tau)$, and $\tau=O\left(L^{-2}\right)$, then the errors between the solutions $\left(u_{d}^{n}, p_{d}^{n}\right)$ to Problem (4.14), and the solutions $\left(u_{h}^{n}, p_{h}^{n}\right)$ to Problem (3.8) have the following error estimates, for $1 \leq n \leq N$ :

$$
\begin{array}{r}
\left\|u_{h}^{n}-u_{d}^{n}\right\|_{0}^{2}+\tau \mathcal{v} \sum_{j=n_{1}}^{n_{i}}\left\|u_{h}^{j}-u_{d}^{j}\right\|_{1}^{2}+\tau \sum_{j=n_{1}}^{n_{i}}\left\|p_{h}^{j}-p_{d}^{j}\right\|_{0}^{2} \leq C \tau^{1 / 2} \sum_{j=d+1}^{l} \lambda_{j}, \\
\text { if } n=n_{i} \in\left\{n_{1}, n_{2}, \ldots, n_{L}\right\}, \\
\left\|u_{h}^{n}-u_{d}^{n}\right\|_{0}^{2}+\tau \mathcal{v}\left[\left\|u_{h}^{n}-u_{d}^{n}\right\|_{1}^{2}+\sum_{j=n_{1}}^{n_{i}}\left\|u_{h}^{j}-u_{d}^{j}\right\|_{1}^{2}\right] \\
+\tau\left[\left\|p_{h}^{n}-p_{d}^{n}\right\|_{0}^{2}+\sum_{j=n_{1}}^{n_{i}}\left\|p_{h}^{j}-p_{d}^{j}\right\|_{0}^{2}\right] \leq C \tau^{1 / 2} \sum_{j=d+1}^{l} \lambda_{j}+C \tau^{2}, \\
\text { if } n=n_{i} \notin\left\{n_{1}, n_{2}, \ldots, n_{L}\right\} .
\end{array}
$$

Proof. Let $w_{d}^{n}=P^{d} u_{h}^{n}-u_{d^{\prime}}^{n} r_{d}^{n}=\rho^{d} p_{h}^{n}-p_{d}^{n}$. Subtracting (3.8) from (4.14) yields that

$$
\begin{aligned}
& \frac{1}{\tau}\left(u_{h}^{n}-u_{d^{\prime}}^{n}, v_{d}\right)-\frac{1}{\tau}\left(u_{h}^{n-1}-u_{d}^{n-1}, v_{d}\right)+a\left(u_{h}^{n}-u_{d^{\prime}}^{n}, v_{d}\right)-b\left(p_{h}^{n}-p_{d^{\prime}}^{n} v_{d}\right) \\
& \quad+b\left(u_{h}^{n-1}-u_{d}^{n-1}, u_{h^{\prime}}^{n} v_{d}\right)+b\left(u_{d}^{n-1}, u_{h}^{n}-u_{d}^{n}, v_{d}\right)+b\left(u_{h}^{n}-u_{d}^{n}, q_{d}\right)+G\left(p_{h}^{n}-p_{d}^{n}, q_{d}\right)=0 .
\end{aligned}
$$

Taking $\left(v_{d}, q_{d}\right)=2 \tau\left(w_{d}^{n}, r_{d}^{n}\right)$ in $(5.4)$, since $a\left(u_{h}^{n}-P^{d} u_{h}^{n}, w_{d}^{n}\right)=0, b\left(u_{h}^{n}-u_{d}^{n}, r_{d}^{n}\right)+G\left(p_{h}^{n}-p_{d}^{n}, r_{d}^{n}\right)=$ 0 , we deduce

$$
\begin{aligned}
& 2\left(w_{d}^{n}, w_{d}^{n}\right)-2\left(w_{d}^{n-1}, w_{d}^{n}\right)+2 \tau a\left(w_{d}^{n}, w_{d}^{n}\right)=2\left(u_{h}^{n-1}-p^{d} u_{h}^{n-1}, w_{d}^{n}\right) \\
& \quad+2\left(p^{d} u_{h}^{n}-u_{h^{\prime}}^{n} w_{d}^{n}\right)+2 \tau b\left(p_{h}^{n}-\rho^{d} p_{h^{\prime}}^{n}, w_{d}^{n}\right)+2 \tau b\left(P^{d} u_{h}^{n-1}-u_{h}^{n-1}, u_{h^{\prime}}^{n} w_{d}^{n}\right) \\
& \quad-2 \tau b\left(w_{d}^{n-1}, u_{h^{\prime}}^{n}, w_{d}^{n}\right)+2 \tau b\left(u_{d}^{n-1}, P^{d} u_{h}^{n}-u_{h^{\prime}}^{n} w_{d}^{n}\right) .
\end{aligned}
$$

Using (2.12)-(2.13), the Hölder inequality and the Young inequality, we see that

$$
\begin{gathered}
\left|\left(u_{h}^{n-1}-p^{d} u_{h}^{n-1}, w_{d}^{n}\right)+\left(p^{d} u_{h}^{n}-u_{h^{\prime}}^{n}, w_{d}^{n}\right)\right| \\
\leq C_{1}\left(\left\|u_{h}^{n-1}-p^{d} u_{h}^{n-1}\right\|_{1}^{2}+\left\|u_{h}^{n}-p^{d} u_{h}^{n}\right\|_{1}^{2}\right)+\frac{v}{10}\left\|w_{d}\right\|_{1}^{2}, \\
\left|b\left(p_{h}^{n}-\rho^{d} p_{h^{\prime}}^{n} w_{d}^{n}\right)\right| \leq C_{4}\left\|p_{h}^{n}-\rho^{d} p_{h}^{n}\right\|_{0}^{2}+\frac{v}{10}\left\|w_{d}^{n}\right\|_{1^{\prime}}^{2} \\
\left|b\left(P^{d} u_{h}^{n-1}-u_{h}^{n-1}, u_{h^{\prime}}^{n} w_{d}^{n}\right)\right| \leq \frac{v}{10}\left\|w_{d}^{n}\right\|_{1}^{2}+C_{5}\left\|\left(u_{h}^{n-1}-P^{d} u_{h}^{n-1}\right)\right\|_{1^{\prime}}^{2} \\
\left|b\left(u_{d}^{n-1}, P^{d} u_{h}^{n}-u_{h^{\prime}}^{n} w_{d}^{n}\right)\right| \leq \frac{v}{10}\left\|w_{d}^{n}\right\|_{1}^{2}+C_{6}\left\|u_{h}^{n}-P^{d} u_{h}^{n}\right\|_{1^{\prime}}^{2} \\
\left|-b\left(w_{d}^{n-1}, u_{h^{\prime}}^{n}, w_{d}^{n}\right)\right| \leq C_{7}\left\|w_{d}^{n-1}\right\|_{0}^{2}+\frac{v}{10}\left\|w_{d}^{n}\right\|_{1}^{2} .
\end{gathered}
$$


Noting that $a(a-b)=\left[a^{2}-b^{2}+(a-b)^{2}\right] / 2$ (for $(a \geq 0$ and $b \geq 0)$ ), owing to (5.6), we obtain that

$$
\begin{aligned}
\left\|w_{d}^{n}\right\|_{0}^{2} & -\left\|w_{d}^{n-1}\right\|_{0}^{2}+v \tau\left\|w_{d}\right\|_{1}^{2} \leq C_{7} \tau\left\|w_{d}^{n-1}\right\|_{0}^{2} \\
& +C \tau\left(\left\|\left(u_{h}^{n}-P^{d} u_{h}^{n}\right)\right\|_{1}^{2}+\left\|\left(u_{h}^{n-1}-P^{d} u_{h}^{n-1}\right)\right\|_{1}^{2}+\left\|p_{h}^{n}-\rho^{d} p_{h}^{n}\right\|_{0}^{2}\right) .
\end{aligned}
$$

First, we consider the case of $n \in\left\{n_{1}, n_{2}, \ldots, n_{L}\right\}$. Summing (5.7) from $n_{1}$ to $n_{i}, i=1,2, \ldots, L$, and noting that $u_{h}^{0}-u_{d}^{0}=0$, using Lemma 4.2 , we can derive that

$$
\left\|w_{d}^{n_{i}}\right\|_{0}^{2}+v \tau \sum_{j=n_{1}}^{n_{i}}\left\|w_{d}^{j}\right\|_{1}^{2} \leq C \tau L \sum_{j=d+1}^{l} \lambda_{j}+C_{7} \tau \sum_{j=n_{0}}^{n_{i-1}}\left\|w_{d}^{j}\right\|_{0}^{2} .
$$

By using the discrete Gronwall inequality, we obtain that

$$
\left\|w_{d}^{n_{i}}\right\|_{0}^{2}+v \tau \sum_{j=n_{1}}^{n_{i}}\left\|w_{d}^{j}\right\|_{1}^{2} \leq C \tau L \sum_{j=d+1}^{l} \lambda_{j} \exp \left(C_{7} \tau n_{i}\right) .
$$

If $h$ and $\tau$ are sufficiently small, $\tau=O\left(L^{-2}\right)$, and noting that $n_{i} \tau \leq n_{i} N \leq T$, we find that

$$
\left\|w_{d}^{n_{i}}\right\|_{0}^{2}+v \tau \sum_{j=n_{1}}^{n_{i}}\left\|w_{d}^{j}\right\|_{1}^{2} \leq C \tau^{1 / 2} \sum_{j=d+1}^{l} \lambda_{j}
$$

Thanks to $b\left(u_{h}^{n}-u_{d}^{n}, r_{d}^{n}\right)+G\left(p_{h}^{n}-p_{d}^{n}, r_{d}^{n}\right)=0$, we obtain

$$
\left\|p_{h}^{n}-p_{d}^{n}\right\|_{0} \leq C\left\|u_{h}^{n}-u_{d}^{n}\right\|_{1}
$$

By combining (5.10)-(5.11) with Lemma 4.2, we obtain the error estimate result (5.2).

Next, we consider the case of $n \notin\{1,2, \ldots, L\}$; we assume that $t_{n} \in\left(t_{n_{i}-1}, t_{n_{i}}\right)$ and $t_{n}$ is the nearest point to $t_{n_{i}} \cdot u_{h}^{n}$ and $p_{h}^{n}$ are expanded into the Taylor series expansion at point $t_{n_{i}}$.

$$
\begin{aligned}
& u_{h}^{n}=u_{h}^{n_{i}}-s \tau \frac{\partial u_{h}\left(\xi_{1}\right)}{\partial t}, \quad \xi_{1} \in\left[t_{n_{i}}, t_{n}\right] \\
& p_{h}^{n}=p_{h}^{n_{i}}-s \tau \frac{\partial p_{h}\left(\xi_{2}\right)}{\partial t}, \quad \xi_{2} \in\left[t_{n_{i}}, t_{n}\right]
\end{aligned}
$$


where $s$ is the number of time steps from $t_{n}$ to $t_{n_{i}}$. If the snapshots are equably taken, then $s \leq N / L$. Summing (5.7) from $n_{1}$ to $n_{i}, \mathrm{n}$, and using (5.12), if $\left|\partial u_{h}\left(\xi_{1}\right) / \partial t\right|$ and $\left|\partial p_{h}\left(\xi_{2}\right) / \partial t\right|$ are bounded, by discrete Gronwall inequality and Lemma 4.2 and (3.12), we obtain that

$$
\left\|w_{d}^{n}\right\|_{0}^{2}+v \tau\left[\left\|w_{d}^{n}\right\|_{1}^{2}+\sum_{j=n_{1}}^{n_{i}}\left\|w_{d}^{j}\right\|_{1}^{2}\right] \leq C \tau L \sum_{j=d+1}^{l} \lambda_{j}+C \tau^{2}
$$

If $\tau=O\left(L^{-2}\right)$, by (5.14) we obtain that

$$
\left\|w_{d}^{n}\right\|_{0}^{2}+v \tau\left[\left\|w_{d}^{n}\right\|_{1}^{2}+\sum_{j=n_{1}}^{n_{i}}\left\|w_{d}^{j}\right\|_{1}^{2}\right] \leq C \tau^{1 / 2} \sum_{j=d+1}^{l} \lambda_{j}+C \tau^{2} .
$$

Hence, combining (5.11), (5.13), and (5.15) with Lemma 4.2 yields (5.3).

Theorem 5.3. Under hypotheses of Theorems 3.4 and 5.2, the error estimates between the solution $(u(t), p(t))$ to Problem (2.1)-(2.2) and the solutions $\left(u_{d^{\prime}}^{n} p_{d}^{n}\right)$ to Problem (4.14) are as follows:

$$
\begin{array}{r}
\left\|u\left(t_{n}\right)-u_{d}^{n}\right\|_{0}^{2}+\tau v \sum_{j=1}^{n}\left\|u^{j}-u_{d}^{j}\right\|_{1}^{2}+\tau \sum_{j=1}^{n}\left\|p^{j}-p_{d}^{j}\right\|_{0}^{2} \\
\leq c\left(h^{2}+\tau^{2}\right)+C \tau^{1 / 2} \sum_{j=d+1}^{l} \lambda_{j}, \quad n=1,2, \ldots, N .
\end{array}
$$

\section{Numerical Examples}

In order to illustrate and verify the theoretical results of Theorem 5.3, we present the results obtained in a simple test case. We set $\Omega$ is the unit square $[0,1] \times[0,1]$ and viscosity $v=0.05$. The velocity and pressure are designed on the same uniform triangulation of $\Omega$. The exact solution is given by

$$
\begin{gathered}
u=\left(u_{1}(x, y), u_{2}(x, y)\right), \quad p(x, y)=10(2 x-1)(2 y-1) \cos (t), \\
u_{1}(x, y)=10 x^{2}(1-x)^{2} y(1-y)(1-2 y) \cos (t), \\
u_{2}(x, y)=-10 x(1-x)(1-2 x) y^{2}(1-y)^{2} \cos (t),
\end{gathered}
$$

and $f$ is determined by (2.1).

All the numerical experiments have been performed using the conforming $Q_{1}$ finite element for both velocity and pressure. The implicit (backward) Euler's scheme is used for the time discretization. For simplicity, the unit square is divided into $n \times n$ small squares with side length $h=1 / n$. In order to make $\tau=O(h)$, we take time step increment as $\tau=1 / n$.

We obtain 20 values (i.e., snapshots) outputting at time $t=10 \tau, 20 \tau, 30 \tau, \ldots, 200 \tau$ by solving the SMFE formulation. We use 7 optimal POD bases to obtain the solutions of the reduced formulation problem (4.14) as $t=200 \tau$. In Table 1 , we present the velocity and 
Table 1: Numerical results for the SMFE method.

\begin{tabular}{cccccc}
\hline$h^{-1}$ & $\frac{\left\|u-u_{h}\right\|_{1}}{\|u\|_{1}}$ & $u_{H^{1}}$ rate & $\frac{\left\|p-p_{h}\right\|_{0}}{\|p\|_{0}}$ & $p_{L^{2}}$ rate & CPU time (seconds) \\
\hline 24 & 0.1443070 & - & 0.0175033 & - & 1081 \\
32 & 0.0973051 & 1.3699 & 0.0104506 & 1.7927 & 2906 \\
40 & 0.0752875 & 1.1497 & 0.0072136 & 1.6612 & 11403 \\
48 & 0.0619347 & 1.0708 & 0.0054000 & 1.5882 & 25375 \\
56 & 0.0510066 & 1.2593 & 0.0042171 & 1.6040 & 42132 \\
64 & 0.0437541 & 1.1486 & 0.0034805 & 1.4375 & 61320 \\
\hline
\end{tabular}

Table 2: Numerical results for the RSMFE method.

\begin{tabular}{cccccc}
\hline$h^{-1}$ & $\frac{\left\|u-u_{d}\right\|_{1}}{\|u\|_{1}}$ & $u_{H^{1}}$ rate & $\frac{\left\|p-p_{d}\right\|_{0}}{\|p\|_{0}}$ & $p_{L^{2}}$ rate & CPU time (seconds) \\
\hline 24 & 0.1486190 & - & 0.0174335 & - & 3 \\
32 & 0.1069230 & 1.1446 & 0.0107643 & 1.6760 & 14 \\
40 & 0.079740 & 1.3146 & 0.0071930 & 1.8066 & 36 \\
48 & 0.0637586 & 1.2268 & 0.0053759 & 1.5971 & 83 \\
56 & 0.0525842 & 1.2500 & 0.0041878 & 1.6202 & 152 \\
64 & 0.0445977 & 1.2337 & 0.0034562 & 1.4379 & 216 \\
\hline
\end{tabular}

pressure relative error estimates, convergence rates, and CPU times using the SMFE method, and, in Table 2, we give the corresponding results obtained using the RSMFE method. In particular, as $n=32$, the SMFE solutions $\left(u_{h i}^{200}, i=1,2\right)(\mathrm{c})$, the exact solutions $\left(u_{i}^{200}, i=1,2\right)$ (b), and the RSMFE solutions $\left(u_{d i}^{200}, i=1,2\right)$ (a) are depicted, respectively, in Figures 1 and 3. Moreover, the difference $\left(u_{i}^{200}-u_{d i}^{200}, i=1,2\right)$ (a) between the exact solutions and the RSMFE solutions and the difference $\left(u_{h i}^{200}-u_{d i}^{200}, i=1,2\right)(\mathrm{b})$ between the SMFE solutions and RSMFE solutions are depicted in Figures 2 and 4, respectively. From Tables 1, 2, and Figures 1-4, we can find that the RSMFE solutions has the same accuracy as the reduced SMFE solutions and the exact solutions. As $n=32$, for the SMFE Problem (3.8)-(3.9), there are $3 \times 32 \times 32=3072$ freedom degrees; the performing time required is 2906 seconds, while the reduced SMFE Problem (4.14) with 7 POD bases only has 7 freedom degrees and the corresponding time is only 14 seconds, that is, the required implementing time to solve the usual SMFE Problem (3.8) is as 207 times as that to do the reduced SMFE problem (4.14) with 7 POD bases, while the errors between their respective solutions do not exceed $3 \times 10^{-3}$. As $n=56$, Figure 5 shows the velocity $H^{1}$ relative errors between solutions with different number of optimal POD bases and solutions obtained with full bases at $t=100 \tau$ and $t=200 \tau$. It is shown that the reduced SMFE problem (4.14) is very effective and feasible. In addition, the results obtained for the numerical examples are consistent with the theoretical ones.

\section{Conclusions}

In this paper, we have combined the POD techniques with a SMFE formulation based on two local Gauss' integrations to derive a reduced SMFE method for the transient NavierStokes equations. The discretization uses a pair of spaces of finite elements $P_{1}-P_{1}$ over triangles or $Q_{1}-Q_{1}$ over quadrilateral elements. This SMFE method differs from that in [45]. It has some prominent features: parameter-free, avoiding higher-order derivatives or 


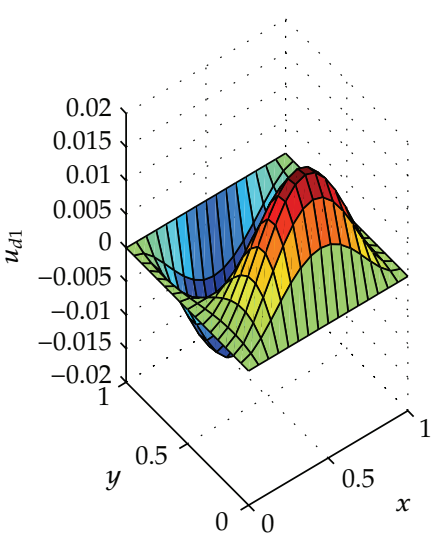

(a)

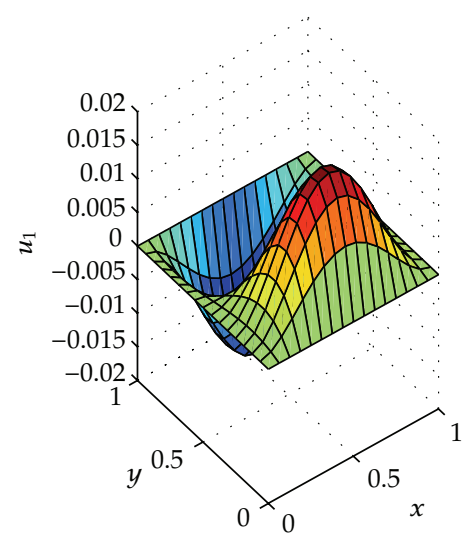

(b)

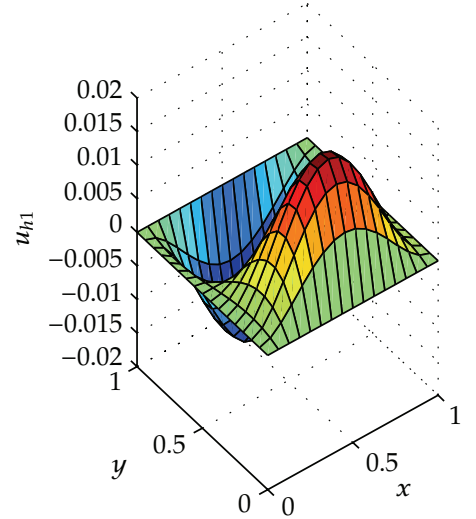

(c)

Figure 1: RSMFE solutions (a), the exact solutions (b), and SMFE solutions (c).

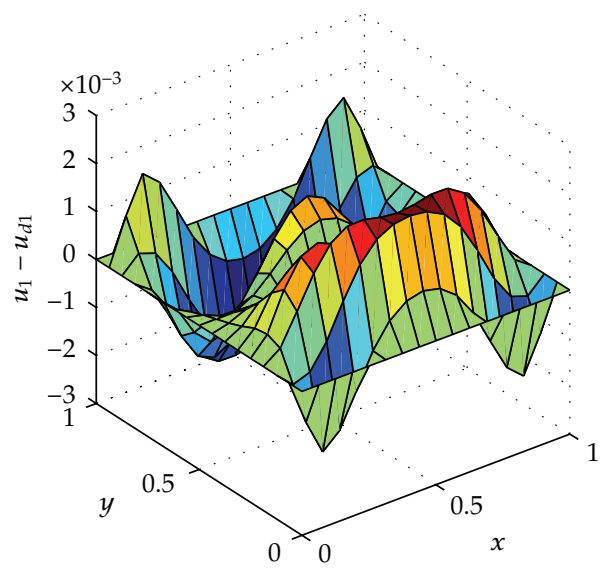

(a)

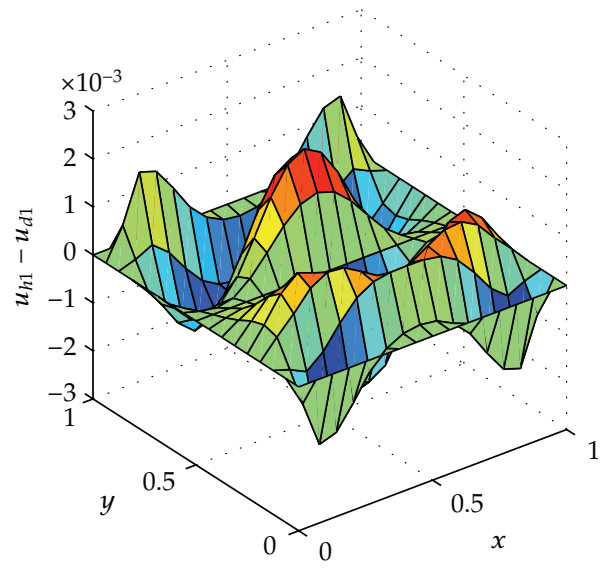

(b)

Figure 2: Difference between the RSMFE solutions and the exact solutions (a) and difference between the SMFE solutions and the RSMFE solutions (b).

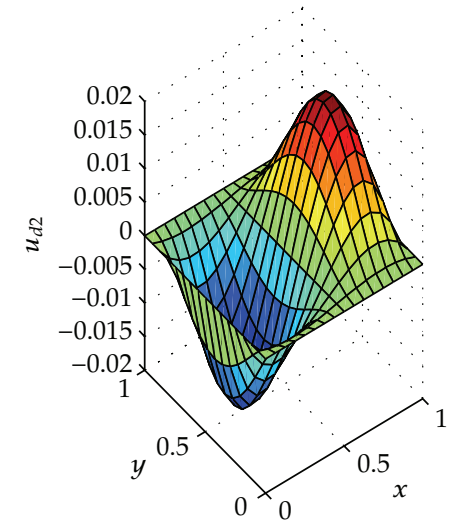

(a)

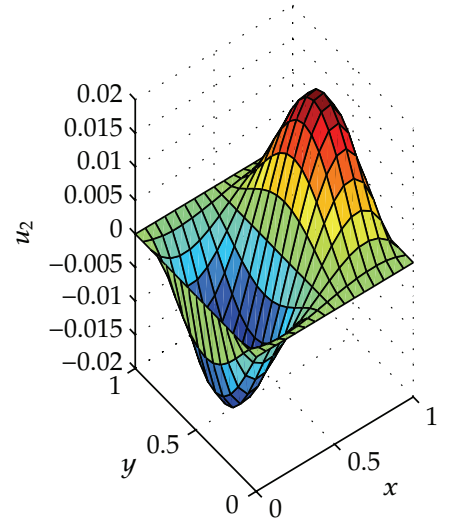

(b)

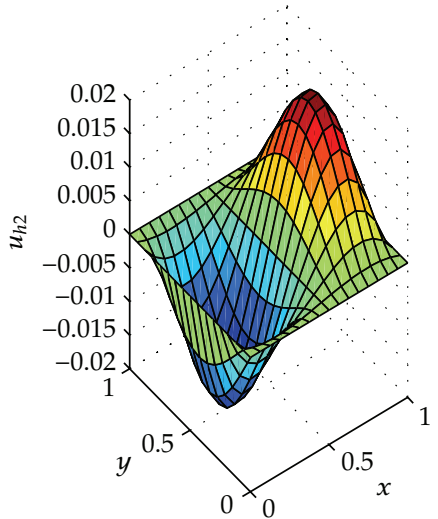

(c)

Figure 3: RSMFE solutions (a), the exact solutions (b), and SMFE solutions (c). 


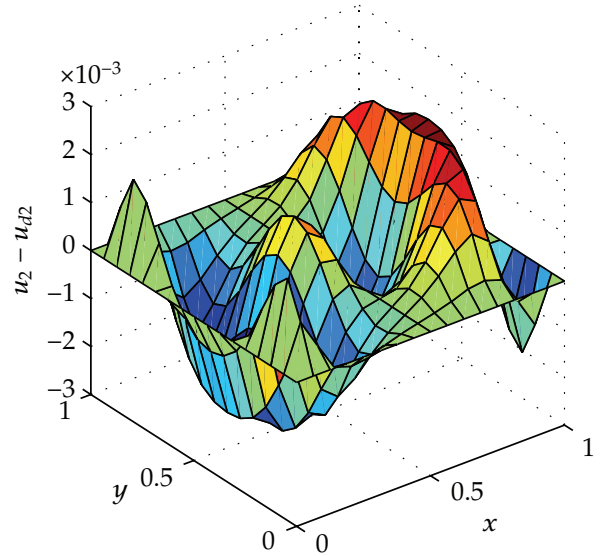

(a)

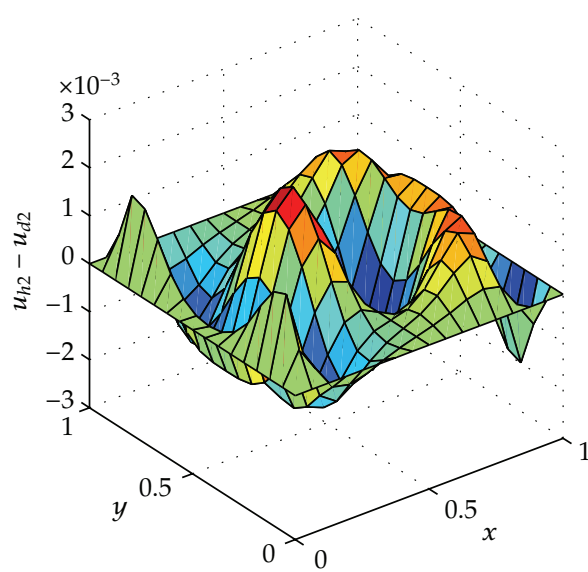

(b)

Figure 4: Difference between the RSMFE solutions and the exact solutions (a) and difference between the SMFE solutions and the RSMFE solutions (b).

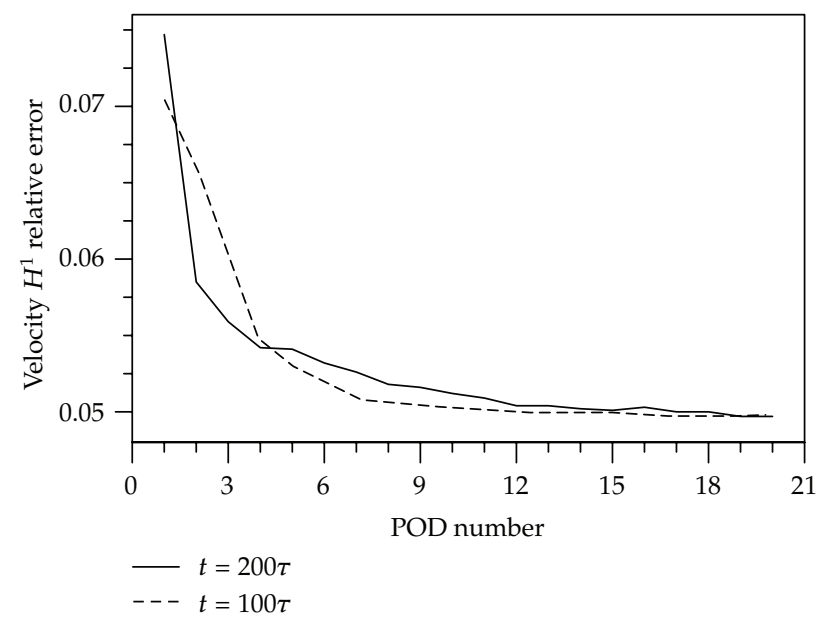

Figure 5: The velocity $H^{1}$-error changing with the number of POD basis as $n=56$.

edge-based data structures, and stabilization being completed locally at the element level. We have also analyzed the errors between the solutions of their usual SMFE formulation and the solutions of the reduced SMFE based on POD basis and discussed theoretically the relation of the number of snapshots and the number of solutions at all time instances, which have shown that our present method has improved and innovated the existing methods. We have validated the correctness of our theoretical results with numerical examples. Though snapshots and the POD basis of our numerical examples are constructed with the solutions of the usual SMFE formulation, when one computes actual problems, one may structure the snapshots and the POD basis with interpolation or data assimilation by drawing samples from experiments, then solve Problem (4.14), while it is unnecessary to solve Problem (3.8). Thus, the time-consuming calculations and resource demands in the computational process are greatly saved, and the computational efficiency is vastly improved. Therefore, the method in this paper holds a good prospect of extensive applications. 
Future research work in this area aims at addressing some practical engineering problems arising in the fluid dynamics and more complicated PDES, extending the optimizing reduced SMFE formulation, applying it to a realistic atmosphere quality forecast system, and to a set of more complicated nonlinear PDES, for instance, 3D realistic model equations coupling strongly nonlinear properties, nonhomogeneous variable flux, and boundary.

\section{Acknowledgments}

This work is supported by the Scientific Research Common Program of the Beijing Municipal Commission of Education (Grant no. KM201110772019), the Knowledge Innovation Program of the Chinese Academy of Sciences (Grant no. KZCX2-EW-QN207), the National Natural Science Foundation of China (Grant no. 11071193 and 41075076), the Academic Human Resources Development in the Institutions of Higher Learning under the Jurisdiction of Beijing Municipality (Grant no. PHR201006116), the Natural Science New Star of Science and Technologies Research Plan in Shaanxi Province of China (Grant no. 2011kjxx12), Research Program of the Education Department of the Shaanxi Province (Grant no. 11JK0490). The author would like to thank the referees for their valuable comments and suggestions.

\section{References}

[1] V. Girault and P.-A. Raviart, Finite Element Methods for Navier-Stokes Equations: Theory and Algorithms, vol. 5 of Springer Series in Computational Mathematics, Springer, New York, NY, USA, 1986.

[2] J. G. Heywood and R. Rannacher, "Finite element approximation of the nonstationary Navier-Stokes problem: I. Regularity of solutions and second-order error estimates for spatial discretization," SIAM Journal on Numerical Analysis, vol. 19, no. 2, pp. 275-311, 1982.

[3] Z. H. Luo, "The third order estimate of mixed finite element for the Navier-Stokes problems," Chinese Quarterly Journal of Mathematics, vol. 10, pp. 9-12, 1995.

[4] K. Fukunaga, Introduction to Statistical Pattern Recognition, Computer Science and Scientific Computing, Academic Press, New York, NY, USA, 2nd edition, 1990.

[5] I. T. Jolliffe, Principal Component Analysis, Springer Series in Statistics, Springer, New York, NY, USA, 2nd edition, 2002.

[6] P. Holmes, J. L. Lumley, and G. Berkooz, Turbulence, Coherent Structures, Dynamical Systems and Symmetry, Cambridge Monographs on Mechanics, Cambridge University Press, Cambridge, UK, 1996.

[7] P. Moin and R. D. Moser, "Characteristic-eddy decomposition of turbulence in a channel," Journal of Fluid Mechanics, vol. 200, pp. 471-509, 1989.

[8] M. Rajaee, S. K. F. Karlsson, and L. Sirovich, "Low-dimensional description of free-shear-flow coherent structures and their dynamical behaviour," Journal of Fluid Mechanics, vol. 258, pp. 1-29, 1994.

[9] R. D. Joslin, M. D. Gunzburger, R. A. Nicolaides, G. Erlebacher, and M. Y. Hussaini, "A self-contained automated methodology for optimal flow control validated for transition delay," AIAA Journal, vol. 35, no. 5, pp. 816-824, 1997.

[10] H. V. Ly and H. T. Tran, "Proper orthogonal decomposition for flow calculations and optimal control in a horizontal CVD reactor," Quarterly of Applied Mathematics, vol. 60, no. 4, pp. 631-656, 2002.

[11] X. J. Tian and Z. H. Xie, "An explicit four-dimensional variational data assimilation method based on the proper orthogonal decomposition: theoretics and evaluation," Science in China D, vol. 52, no. 2, pp. 279-286, 2009.

[12] J. Lumley, "Coherent structures in turbulence," in Transition and Turbulence, R. E. Meyer, Ed., Academic Press, New York, NY, USA, 1981.

[13] N. Aubry, P. Holmes, J. L. Lumley, and E. Stone, "The dynamics of coherent structures in the wall region of a turbulent boundary layer," Journal of Fluid Mechanics, vol. 192, pp. 115-173, 1988.

[14] L. Sirovich, "Turbulence and the dynamics of coherent structures: part I-III," Quarterly of Applied Mathematics, vol. 45, no. 3, pp. 561-590, 1987.

[15] K. Kunisch and S. Volkwein, "Galerkin proper orthogonal decomposition methods for parabolic problems," Numerische Mathematik, vol. 90, no. 1, pp. 117-148, 2001. 
[16] K. Kunisch and S. Volkwein, "Galerkin proper orthogonal decomposition methods for a general equation in fluid dynamics," SIAM Journal on Numerical Analysis, vol. 40, no. 2, p. 492-515 (electronic), 2002.

[17] Z. D. Luo, R. W. Wang, and J. Zhu, "Finite difference scheme based on proper orthogonal decomposition for the nonstationary Navier-Stokes equations," Science in China A, vol. 50, no. 8, pp. 1186-1196, 2007.

[18] Z. D. Luo, J. Chen, I. M. Navon, and X. Z. Yang, “Mixed finite element formulation and error estimates based on proper orthogonal decomposition for the nonstationary Navier-Stokes equations," SIAM Journal on Numerical Analysis, vol. 47, no. 1, pp. 1-19, 2008.

[19] Z. D. Luo, J. Chen, P. Sun, and X. Z. Yang, "Finite element formulation based on proper orthogonal decomposition for parabolic equations," Science in China A, vol. 52, no. 3, pp. 585-596, 2009.

[20] Z. D. Luo, X. Z. Yang, and Y. J. Zhou, "A reduced finite element formulation based on proper orthogonal decomposition for Burgers equation," Applied Numerical Mathematics, vol. 59, no. 8, pp. 1933-1946, 2009.

[21] Y. H. Cao, J. Zhu, Z. D. Luo, and I. M. Navon, "Reduced-order modeling of the upper tropical Pacific Ocean model using proper orthogonal decomposition," Computers \& Mathematics with Applications, vol. 52, no. 8-9, pp. 1373-1386, 2006.

[22] Y. H. Cao, J. Zhu, I. M. Navon, and Z. D. Luo, "A reduced-order approach to four-dimensional variational data assimilation using proper orthogonal decomposition," International Journal for Numerical Methods in Fluids, vol. 53, no. 10, pp. 1571-1583, 2007.

[23] Z. D. Luo, J. Zhu, R. W. Wang, and I. M. Navon, “Proper orthogonal decomposition approach and error estimation of mixed finite element methods for the tropical Pacific Ocean reduced gravity model," Computer Methods in Applied Mechanics and Engineering, vol. 196, no. 41-44, pp. 4184-4195, 2007.

[24] Z. D. Luo, J. Chen, J. Zhu, R. W. Wang, and I. M. Navon, "An optimizing reduced order FDS for the tropical Pacific Ocean reduced gravity model," International Journal for Numerical Methods in Fluids, vol. 55, no. 2, pp. 143-161, 2007.

[25] R. W. Wang, J. Zhu, Z. D. Luo, and I. M. Navon, “An equation-free reduced order modeling approach to tropic Pacific simulation," Advances in Geosciences, vol. 12, pp. 1-16, 2007.

[26] J. Du, J. Zhu, Z. D. Luo, and I. M. Navon, "An optimizing finite difference scheme based on proper orthogonal decomposition for CVD equations," International Journal for Numerical Methods in Biomedical Engineering, vol. 27, no. 1, pp. 78-94, 2011.

[27] H. L. Yu and H. J. Chu, “Understanding space-time patterns of groundwater system by empirical orthogonal functions: a case study in the Choshui River alluvial fan, Taiwan," Journal of Hydrology, vol. 381, no. 3-4, pp. 239-247, 2010.

[28] T. Bernard, O. Krol, H. Linke, and T. Rauschenbach, "Optimal management of regional water supply systems using a reduced finite-element groundwater model," Automatisierungstechnik, vol. 57, no. 12, pp. 593-600, 2009.

[29] J. Li and Y. N. He, "A stabilized finite element method based on two local Gauss integrations for the Stokes equations," Journal of Computational and Applied Mathematics, vol. 214, no. 1, pp. 58-65, 2008.

[30] J. Li, Y. N. He, and Z. X. Chen, "A new stabilized finite element method for the transient Navier-Stokes equations," Computer Methods in Applied Mechanics and Engineering, vol. 197, no. 1-4, pp. 22-35, 2007.

[31] J. Li, Finite element methods for the two-dimensional impressible Navier-Stokes equations, Ph.D. thesis, Xi'an Jiaotong University, 2007.

[32] S. Li and Y. R. Hou, "A fully discrete stabilized finite element method for the time-dependent NavierStokes equations," Applied Mathematics and Computation, vol. 215, no. 1, pp. 85-99, 2009.

[33] P. B. Bochev, C. R. Dohrmann, and M. D. Gunzburger, "stabilization of low-order mixed finite elements," SIAM Journal on Numerical Analysis, vol. 42, pp. 1189-1208, 2006.

[34] A. W. Wang and J. Li, "Stabilization of the lowest-order mixed finite elements for the steady NavierStokes equations," Chinese Journal of Engineering Mathematics, vol. 17, pp. 249-257, 2010.

[35] Y. N. He, A. W. Wang, and L. Q. Mei, "Stabilized finite-element method for the stationary NavierStokes equations," Journal of Engineering Mathematics, vol. 51, no. 4, pp. 367-380, 2005.

[36] T. J. R. Hughes, L. P. Franca, and M. Balestra, "A new finite element formulation for computational fluid dynamics: V. Circumventing the Babuska-Brezzi condition: a stable Petrov-Galerkin formulation of the Stokes problem accommodating equal-order interpolations," Computer Methods in Applied Mechanics and Engineering, vol. 59, no. 1, pp. 85-99, 1986. 
[37] T. J. R. Hughes and L. P. Franca, "A new finite element formulation for computational fluid dynamics: VII. The Stokes problem with various well-posed boundary conditions: symmetric formulations that converge for all velocity/pressure spaces," Computer Methods in Applied Mechanics and Engineering, vol. 65, no. 1, pp. 85-96, 1987.

[38] J. Douglas and J. P. Wang, "An absolutely stabilized finite element method for the Stokes problem," Mathematics of Computation, vol. 52, no. 186, pp. 495-508, 1989.

[39] C. Johnson and J. Saranen, "Streamline diffusion methods for the incompressible Euler and NavierStokes equations," Mathematics of Computation, vol. 47, no. 175, pp. 1-18, 1986.

[40] B. Kragel, Streamline diffusion POD models in optimization, Ph.D. thesis, Universitat Trier, 2005.

[41] P. Hansbo and A. Szepessy, "A velocity-pressure streamline diffusion finite element method for the incompressible Navier-Stokes equations," Computer Methods in Applied Mechanics and Engineering, vol. 84, no. 2, pp. 175-192, 1990.

[42] M. A. Behr, L. P. Franca, and T. E. Tezduyar, "Stabilized finite element methods for the velocitypressure-stress formulation of incompressible flows," Computer Methods in Applied Mechanics and Engineering, vol. 104, no. 1, pp. 31-48, 1993.

[43] J. Blasco and R. Codina, "Space and time error estimates for a first order, pressure stabilized finite element method for the incompressible Navier-Stokes equations," Applied Numerical Mathematics, vol. 38, no. 4, pp. 475-497, 2001.

[44] R. Codina and J. Blasco, "Analysis of a pressure-stabilized finite element approximation of the stationary Navier-Stokes equations," Numerische Mathematik, vol. 87, no. 1, pp. 59-81, 2000.

[45] Z. D. Luo, J. Du, Z. Xie, and Y. Guo, "A reduced stabilized mixed finite element formulation based on proper orthogonal decomposition for the non-stationary Navier-Stokes equations," International Journal for Numerical Methods in Engineering, vol. 88, no. 1, pp. 31-46, 2011.

[46] R. Temam, Navier-Stokes Equations: Theory and Numerical Analysis, vol. 2 of Studies in Mathematics and its Applications, North-Holland, Amsterdam, The Netherlands, 3rd edition, 1984. 


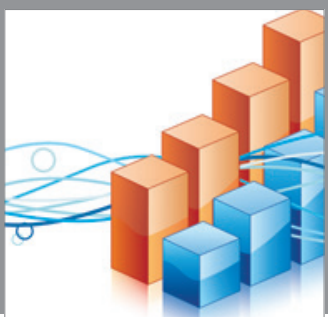

Advances in

Operations Research

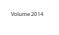

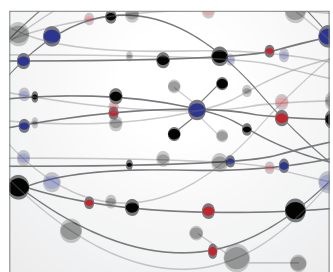

\section{The Scientific} World Journal
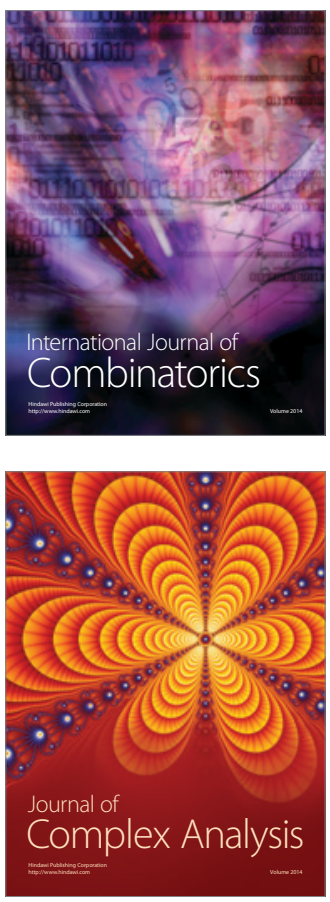

International Journal of

Mathematics and

Mathematical

Sciences
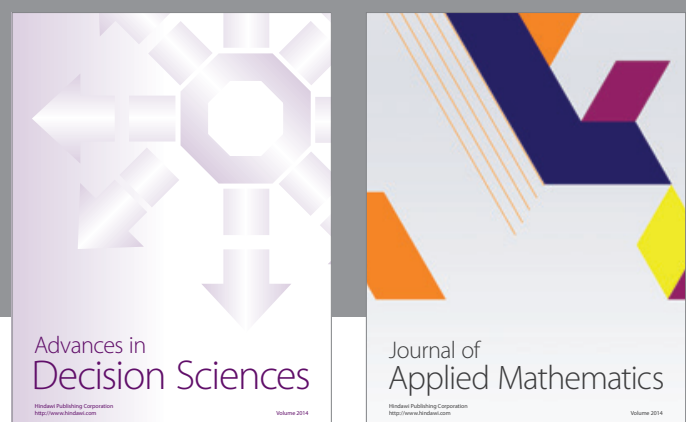

Journal of

Applied Mathematics
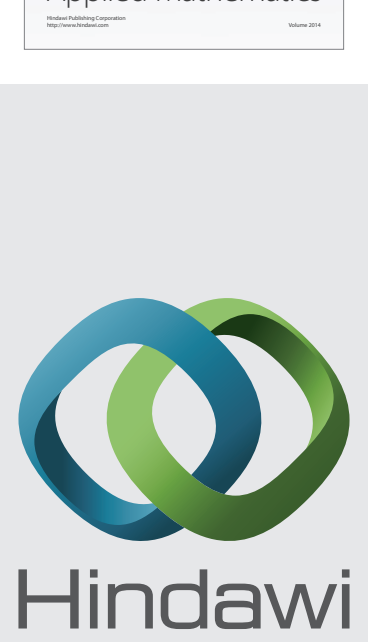

Submit your manuscripts at http://www.hindawi.com
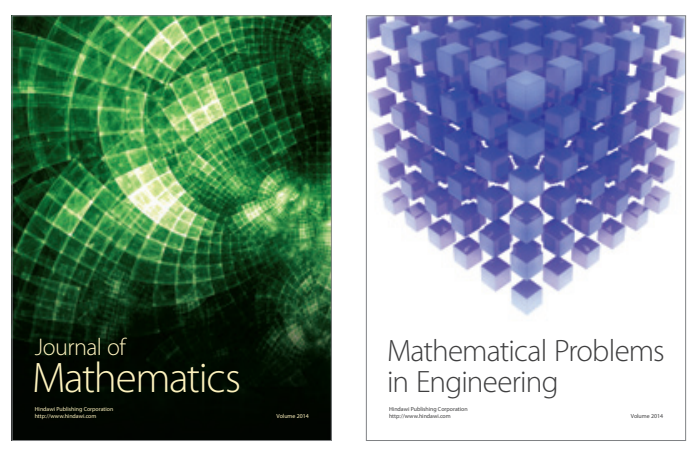

Mathematical Problems in Engineering
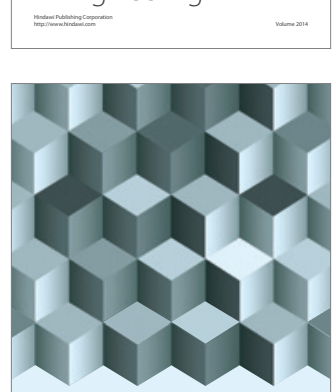

Journal of

Function Spaces
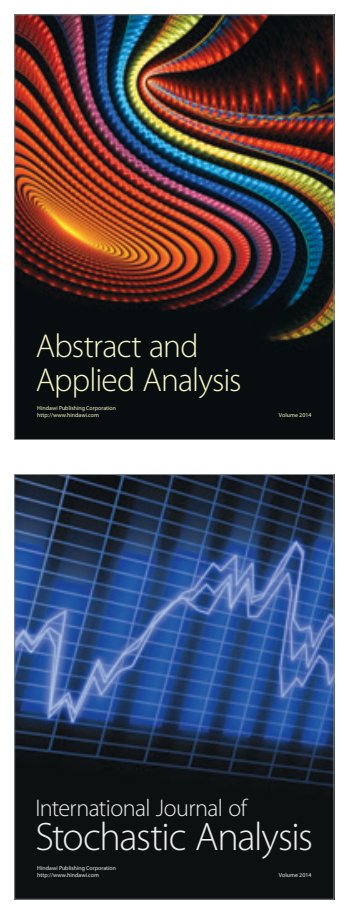

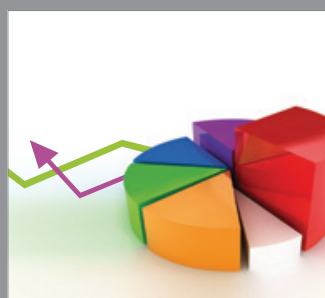

ournal of

Probability and Statistics

Promensencen
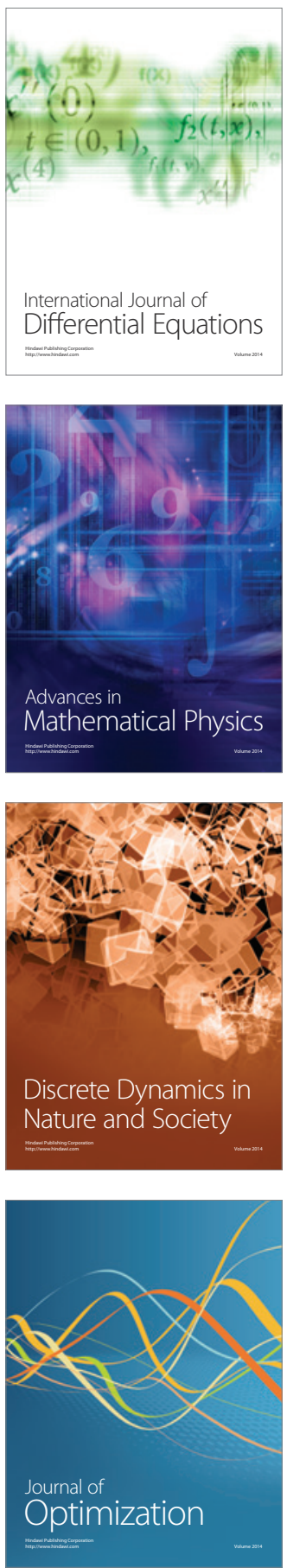